\title{
Coordinación para la intervención en los problemas de comunicación de niños con necesidades de at: un estudio preliminar de necesidades
}

\section{Coordination for intervention in the communication problems of children with at needs: a preliminary needs assessment}

DOI: $10.46932 / \mathrm{sfjdv2n5-018}$

Received in: Jun 1st, 2021

Accepted in: Sep 30th, 2021

\author{
Alba Rodríguez Capdepón \\ Maestra de Educación Infantil. Universidad de Murcia \\ Ángela Pérez Lencina \\ Maestra de Educación Infantil. Universidad de Murcia \\ María Moreno Ruiz \\ Maestra de Educación Infantil. Universidad de Murcia
}

\begin{abstract}
RESUMEN
En el ámbito de la Atención Temprana, existe la discusión en cuanto al nivel y la calidad de la coordinación existente entre los principales implicados en los procesos de intervención. Es por ello, que el principal objetivo de este artículo es analizar el nivel y la calidad de coordinación entre los centros de Atención Temprana, los centros de Educación Infantil y las familias que tienen un niño con problemas de comunicación para cuya mejora puede ser conveniente implementar un sistema aumentativo de comunicación. Para poder llevar a cabo este artículo de investigación, se ha elaborado un estudio de enfoque mixto exploratorio secuencial, con el propósito de obtener una complementariedad paradigmática y metodológica. Se ha realizado una búsqueda empírica sobre la bibliografía que trata esta problemática, y después se ha elaborado el planteamiento de tres métodos de investigación de enfoque cualitativo y cuantitativo. Se ha elaborado una aproximación de los resultados que podrían haberse obtenido de haber llevado a cabo la práctica de investigación, y se han obtenido unas conclusiones al respecto. La situación de Covid19 que estamos atravesando ha impedido la puesta en práctica de esta investigación, llevándonos a plantear posibles escenarios que podrían haberse dado de haber sido posible la realización de este artículo. Sin embargo, hemos podido concluir que existe una coordinación entre estos agentes implicados, pero es demasiado escasa y no se da con la frecuencia que debería, y, por lo tanto, sería conveniente tener en cuenta esta insuficiente coordinación para posibles futuras intervenciones.
\end{abstract}

Palabras clave: Familia, Coordinación de la Educación, Psicología, Comunicación, Educación de la primera infancia.

\begin{abstract}
In the field of Early Intervention, there is a controversy regarding the level and quality of the coordination between the main members involved in the intervention processes. For this reason, the main objective of this work is to analyze the level and quality of coordination between Early Intervention centers, Early Childhood Education centers, and families who have a child with communication problems, for whose improvement it may be convenient to implement an augmentative communication system. In order to carry out this research work, a sequential exploratory mixed approach study has been developed, with the purpose of obtaining a paradigmatic and methodological complementarity. We have done an empirical research on the bibliography that deals with this problem, and then, the approach of three research methods
\end{abstract}


with a qualitative and quantitative approach has been elaborated. We have done an approximation of the results that could have been obtained from having carried out the research practice, and some conclusions have been obtained in this regard. The Covid19 situation that we are going through has made it impossible to put this investigation into practice, leading us to propose possible scenarios that could have occurred had it been possible to carry out this work. However, we have concluded that there is coordination between these agents involved, but it is too scarce and does not occur as often as it should, and, therefore, it would be convenient to take into account this insufficient coordination for possible future interventions.

Keywords: Family, Educational coordination, Psychology, Communication, Early childhood education.

\section{INTRODUCCIÓN}

Hoy en día, la Atención Temprana parece ser un concepto novedoso y que está en boca de todos, ya que es utilizado en muchos ámbitos con el fin de atenuar o disminuir secuelas o efectos que pueden derivarse de distintas enfermedades o trastornos. Además, no solo niños con algún tipo de problema diagnosticado pueden acudir a centros de Atención Temprana, sino que muchas familias optan por asistir con sus hijos por diversos motivos, como pueden ser retrasos motores o del habla por inmadurez, entre otras muchas cuestiones. Sin embargo, la idea de la atención precoz en niños con necesidades surgió muchos años atrás.

\section{MARCO EMPÍRICO}

\section{OBJETIVOS}

\section{Objetivo principal}

- Analizar el nivel y la calidad de coordinación entre los centros de Atención Temprana, los centros de Educación Infantil y las familias que tienen un niño con problemas de comunicación para cuya mejora puede ser conveniente implementar un sistema aumentativo de comunicación.

\section{Objetivos específicos}

- Contrastar con los diferentes implicados las posibilidades y necesidades de coordinación a partir de sus experiencias vividas a través de procedimientos de investigación de enfoque cualitativo.

- Comparar las diferencias acerca del nivel de coordinación que se da en centros concertadosprivados y centros públicos con las familias y profesionales de Atención Temprana que tienen un niño con problemas de comunicación.

- Elaborar una serie de resultados esperados para este estudio. 


\subsection{METODOLOGÍA}

\subsubsection{Enfoque y Diseño}

Para este estudio, se llevarán a cabo tres procedimientos de recogida de información, con distintas muestras, para lograr un groso de información ecléctica que nos permita comparar y contrastar la información. Es por ello, que se puede argumentar que utilizaremos un enfoque mixto para la elaboración de dicho artículo, combinando enfoque cualitativo y cuantitativo, con el fin de obtener una complementariedad paradigmática y metodológica. En concreto, se realizará un estudio mixto de diseño exploratorio secuencial, en el cual se recogerán y analizarán los datos cualitativos, con el fin de recoger y analizar más tarde los datos cuantitativos, y poder realizar una interpretación de los mismos de una forma más completa.

La primera parte de este estudio se fundamenta en un diseño cualitativo de tipo fenomenológico, ya que procuraremos tratar de entender experiencia de un grupo de personas en cuanto a la coordinación que existe entre los distintos sujetos entrevistados y participantes expertos en el grupo focal, con respecto a la asistencia de infantes a un CDIAT con el fin de implementar un sistema alternativo de comunicación.

La segunda parte de este estudio se basará en un estudio cuantitativo de tipo descriptivo, con el cual pasaremos un cuestionario o encuesta a una muestra más amplia que la utilizada en la primera parte de nuestro estudio, con el fin de conocer un baremo más realista sobre el nivel y la calidad de coordinación que se da entre los distintos colectivos seleccionados, con respecto a la implementación de un sistema alternativo de comunicación en niños, en un CDIAT.

En primer lugar, se realizarán una serie de entrevistas tanto a familias, en concreto uno de los tutores legales del niño, así como a profesionales de Atención Temprana y maestros tutores del centro educativo donde acude el niño, en un contexto donde tenemos constancia de que sí existe una coordinación entre estos individuos, con el fin de conocer cuál es el nivel y la calidad de esta comunicación, y tener una guía sobre qué se debe hacer para que exista esta organización entre los colectivos, para poder, después procesar la información y organizar los grupos de discusión en base a esta información.

Tras esto, se organizarán unos grupos de discusión, donde tanto familiares, como profesionales de AT y maestros interactuarán a través de unas preguntas guiadas que se irán enunciando, con el objetivo de conocer distintas opiniones, percepciones y experiencias acerca de la implementación de SAACs y de la coordinación que existe o no entre los múltiples ejes que se encuentran implicados en dicha intervención, cómo debería darse esa coordinación, y por qué es o no esencial para el desarrollo del infante, entre otros aspectos que pueden ir surgiendo a lo largo de todo el debate. Los resultados obtenidos de este grupo de discusión, nos servirán, una vez procesados, para la elaboración de un cuestionario que se pasará a una muestra más amplia. 
Finalmente, realizaremos un cuestionario, el cual pasará por un juicio de expertos y por una aplicación piloto antes de llegar finalmente a su muestra real y válida. Esta muestra constará de padres o familiares, profesionales de AT y maestros que se encuentren en contacto con niños que necesitan de un sistema alternativo o aumentativo de comunicación. A partir de este, se extraerán unos resultados que, una vez procesados, podremos interpretar a modo de conclusiones del estudio empírico.

Teniendo en cuenta la situación actual que estamos atravesando, este estudio será una investigación ficticia, la cual será planteada, pero no llevada a cabo.

\subsubsection{Fase 1. Entrevista.}

Participantes y contexto. Tal y como hemos mencionado en el apartado anterior, la primera fase consta de una entrevista que realizaremos a una pequeña muestra. Esta muestra ha sido escogida de un modo no probabilístico, de forma intencional, ya que se requería para este estudio que esta primera muestra fuera un colectivo de personas en cuyo entorno sí se dé una buena coordinación entre los padres, el profesional de AT y el maestro del niño con problemas de comunicación y lenguaje. Se ha decidido que así sea, puesto que esto nos ayudará a realizar una clasificación sobre las buenas prácticas que deben seguirse para conseguir que exista esta organización entre los miembros de los que depende el niño, qué métodos o técnicas han seguido para conseguirlo y cuál ha sido su experiencia al ponerlo en práctica.

Por lo tanto, en las entrevistas iniciales, se ha escogido intencionalmente a uno de los padres de un niño de 4 años con Trastorno del Espectro Autista, el cual acude a un centro de Atención Temprana en Murcia, dos veces a la semana, durante el curso escolar, es decir, desde septiembre hasta junio. Este niño presenta dificultades en la comunicación y lenguaje, se encuentra en proceso de implementación de un sistema de comunicación aumentativo en el mismo centro. Además de este, participará el profesional de AT de este mismo centro, el cual es el principal responsable del niño mientras se encuentra en el centro, pone en práctica este SAAC y puede comprobar su progreso y desarrollo. Por último, también será entrevistado el maestro tutor del centro educativo al que asiste el niño, quien se encuentra al tanto de la implementación de este SAAC y pone en práctica en su aula distintos métodos que le han sido transmitidos desde el CDIAT para procurar ser lo más fiel posible a la intervención mencionada. Se contactó con estas personas a través de vía telefónica, con el fin de confirmar su asistencia y concretar el momento y el lugar de la cita.

En cuanto al contexto de la muestra perteneciente a esta fase del estudio, las tres personas viven en la ciudad de Murcia. El nivel de estudios de los dos profesionales participantes es universitario, mientras que el progenitor entrevistado cuenta con estudios de formación profesional. 
Diseño de la Intervención. Para la realización de la primera fase de este artículo de investigación, han sido elaboradas tres entrevistas ad hoc dirigidas a las tres personas seleccionadas en la muestra de forma no probabilística.

Para ello, se ha tenido en cuenta el papel que juegan en el desarrollo del niño, la existente coordinación que existe entre los tres colectivos y la cual tenemos constancia de ello, así como la bibliografía que ha sido estudiada y analizada anteriormente. (Anexo 1). Además, también se ha tenido en cuenta el tipo de trastorno que posee el niño, quien se encuentra en un proceso de intervención para la implementación de un sistema de comunicación alternativo, con el fin de propiciar un desarrollo del lenguaje del niño a través de otra vía que no es la tradicional, ya que esta no ha tenido los efectos esperados.

Las preguntas de estas entrevistas están orientadas a dar respuesta conocer cuál es el nivel de coordinación que se da en este ámbito, cómo han hecho estos tres ejes para conseguir dicha organización y propiciar unos buenos resultados con la misma, cuál es la calidad de esta coordinación, ventajas e inconvenientes que pueden encontrarse y qué aspectos se podrían mejorar de estas intervenciones.

Recogida de Información: Instrumentos y Procedimientos. Las entrevistas elaboradas para este de investigación han sido creadas ad hoc, teniendo en cuenta las características de la muestra, así como toda la bibliografía seleccionada y analizada con anterioridad. Las entrevistas constan de 9-10 preguntas cada una, de una extensión media.

Estas entrevistas serán realizadas de forma presencial, cuidando las medidas de seguridad para el protocolo de Covid19, de modo que se disfrute de un entorno seguro y agradable. De ser posible, las entrevistas serán realizadas durante la primera semana que dura el estudio, con la intención de obtener los resultados y poder analizarlos para llevar a cabo el siguiente procedimiento, es decir, los grupos de discusión. En la entrevista (Anexo 1), se muestran las instrucciones que se le dan a las personas entrevistadas, con el fin de que la intervención sea lo más fructífera posible.

Se prevé que cada una de las entrevistas tenga una duración de entre 40 y 45 minutos, ya que se llevarán a cabo de forma oral y serán grabadas, para después poder realizar una transcripción de las mismas, y poder analizar los datos con más seguridad y firmeza.

Tras la transcripción de estas entrevistas, nos tomará unas dos semanas el análisis de la información, así como la organización de los grupos de discusión en base a los resultados que hemos obtenido en dichas entrevistas.

Plan de Tratamiento y Análisis de los Resultados. Con el fin de agilizar el proceso de análisis de la información obtenida, se utilizaría el programa Atlas.ti, ya que se trata de una herramienta muy útil a la hora de analizar la información de investigaciones de tipo cualitativo. Según Muñoz (2017), este 
software nos permite realizar una serie de funciones con los datos obtenidos en un estudio cualitativo, como pueden ser "gestionar grandes volúmenes de datos, almacenar de forma organizada la información elaborada durante el análisis, segmentar, codificar y recuperar fragmentos significativos de nuestro material empírico y elaborar anotaciones del proceso y los resultados del análisis.” (p. 4).

Como hemos mencionado en el apartado anterior, estas entrevistas quedarán grabadas, a través de una grabadora de audio de la marca SONY IC Recorder, modelo ICD-UX200, con disco interno de 2GB, con el fin de poder recuperar el máximo de detalles que nos sea posible. A los participantes, previo a la grabación de las entrevistas, se les otorgará un consentimiento informado (Anexo 5), informándoles sobre el anonimato de los sujetos en todo momento y el tratamiento de los datos. Además, la entrevistadora también contará con una hoja de anotaciones donde irá escribiendo temas de relevancia que puedan ir surgiendo durante la entrevista.

Una vez transcritas las tres entrevistas realizadas, procederemos a introducir los datos al programa informático. En primer lugar, crearemos una nube de palabras que represente los términos que más se repiten en dichas entrevistas. Tras esto, se extraerán los conceptos o tópicos que más se repiten a lo largo de las mismas, de modo que creemos en el programa unos códigos que más tarde nos permitirán enlazar toda la información, y así poder ver las conexiones que existen entre las distintas entrevistas. Finalmente, se procederá a crear las redes, en función de los objetivos de investigación en las cuales están basadas las preguntas de las entrevistas, y hemos especificado con anterioridad. De este modo, se podrá examinar con facilidad qué opina cada interlocutor con respecto a los tópicos más repetidos durante las entrevistas y se podrán analizar con exactitud los resultados obtenidos.

Estos resultados servirán de base para la organización de la siguiente fase de este estudio de investigación, la cual está basada en grupos de discusión. La información obtenida nos permitirá estructurar las distintas cuestiones a debatir, de modo que esto permita percibir las distintas opiniones dentro de colectivos semejantes con respecto a la coordinación en cuanto a la implementación de sistemas de comunicación alternativos y/o aumentativos.

Resultados. Si bien recordamos, este informe de investigación se trata de un planteamiento y formulación de una posible investigación ficticia, por lo que no se ha podido recoger una información real acerca de las entrevistas que han sido elaboradas ad hoc para dicho estudio empírico. Es por ello, y por la información que hemos argumentado más arriba, que el apartado de resultados se limitará a una redacción de los posibles resultados que se podrían haber obtenido de los encuentros que se han dado con los colectivos entrevistados, teniendo en cuenta que se trabaja con una muestra no probabilística intencional con el objetivo de que esta fuera un grupo de personas entre los que sí se da una coordinación a la hora de implementar un sistema de comunicación alternativo y/o aumentativo. 
Tras la recogida y el análisis de la información que se ha obtenido tras llevar a cabo las entrevistas pertinentes a esta primera fase de la investigación, se puede afirmar que se da una coordinación media entre los distintos colectivos implicados.

Como se ha podido comprobar, el progenitor del niño asiste a cada una de las reuniones concertadas con el profesional de AT en el centro. Estas reuniones suelen darse, por lo general, una vez al mes. Sin embargo, en caso de ser necesario debido a algún tipo de interrupción en el proceso, se afirma que no hay problema en hacer reuniones más a menudo.

En cuanto a las reuniones del padre y el maestro, ambos afirman que son los dos progenitores los que acuden a dichos encuentros, con el fin de obtener más información acerca del transcurso de su hijo a lo largo del curso escolar. Estas reuniones suelen realizarse al principio y al final de cada trimestre, con el objetivo de sentar las bases del seguimiento que se le está haciendo al infante tanto en casa como en el CDIAT.

Finalmente, entre el maestro y el profesional de AT solo se da comunicación vía email o por teléfono, pero no se dan encuentros presenciales. A pesar de ello, ambos afirman que es suficiente para realizar el seguimiento que se requiere al niño que se está interviniendo, y que no habría problema a la hora de concertar una reunión presencial, si la situación lo requiere.

En cuanto a la calidad de dichas reuniones, tal y como afirma el padre del niño, se tratan distintas cuestiones. En primer lugar, se aclaran y solucionan dudas que van surgiendo a lo largo del mes en el cual el niño acude al centro, como hemos dicho antes, dos veces por semana. Además, el profesional de AT le proporciona pautas a la familia para seguir la intervención desde casa, en el entorno natural del niño. Ambos sujetos afirman que las técnicas son transmitidas con claridad y precisión, con el fin de que sea lo más fiel posible al modelo que se sigue desde el CDIAT.

Además, en estos encuentros, también se tiene en cuenta la experiencia de los padres en casa, poniendo en práctica dichas técnicas. Según declara el profesional de AT, solicita a los progenitores que se excedan en detalles sobre la aplicación que hacen del SAAC en casa y en otros entornos donde el niño interacciona con sus iguales o con otros adultos que conoce, como pueden ser reuniones de amigos, el centro educativo o actividades extraescolares donde acude el niño con problemas de comunicación.

Asimismo, el profesional de AT y el maestro argumentan que se encuentran en constante contacto mediante el correo electrónico, y a través de este, comparten herramientas que pueden serles útiles a la hora de la implementación del SAAC. Según afirma el maestro entrevistado, más bien es el profesional de AT el que le comparte estas herramientas mencionadas, con el objetivo de que sean utilizadas y las ponga en práctica en el aula con este alumno, y es el maestro el que le retorna estas fichas o ejercicios, de 
modo que se da un feedback entre ambos sujetos y se tiene constancia en sendos centros del desarrollo y posible progreso del niño con dificultades de comunicación y lenguaje.

A continuación podemos ver una figura (Figura 3) que resume los resultados obtenidos en las entrevistas que han sido realizadas.

Discusión de los Resultados. Para valorar los resultados y poder discutirlos nos guiaremos por los objetivos iniciales del estudio.

El primero de nuestros objetivos consistía en analizar el nivel y la calidad de coordinación entre los centros de Atención Temprana, los centros de Educación Infantil y las familias que tienen un niño con problemas de comunicación para cuya mejora puede ser conveniente implementar un sistema aumentativo de comunicación.

Tras la realización de las entrevistas, la transcripción de las mismas y el análisis de los datos obtenidos, podríamos decir que sí se da una coordinación entre los distintos agentes implicados en el estudio.

Tanto los padres del niño que precisa de Atención Temprana, y se encuentra en un proceso de implementación de un SAAC, así como el profesional de AT y el maestro-tutor del mismo, son conocedores del tipo de sistema alternativo de comunicación que está siendo implementado, y han recibido información desde el CDIAT sobre lo que es un SAAC y los distintos tipos que existen.

Por un lado, el progenitor entrevistado argumenta que sí se dan reuniones de calidad con el profesional de AT. Tal y como podemos observar en la bibliografía revisada, es crucial tener en cuenta a la familia del niño que está participando de la intervención, puesto que se trata de su entorno más cercano, y donde el niño se desenvuelve de forma más natural. No se puede aislar esta intervención al centro de Atención Temprana, ya que este no es un entorno real para el niño, sino que sabe que está siendo intervenido, y que, una vez abandone el centro, no se encuentra con profesionales que le den las pautas necesarias. Es por ello, que desde el primer momento de la intervención se ha de hacer saber a la familia cómo han de actuar en todo momento para que la implementación de un sistema de comunicación aumentativo o, en este caso, alternativo, sea fructífero y dé los resultados que se pretenden.

En este caso, la familia se encuentra totalmente implicada en todo el proceso de actuación, y se sienten parte del mismo. Como hemos visto más arriba, esto es algo imprescindible, ya que tanto la familia como el profesional de AT van a sentir la seguridad de estar actuando de forma correcta frente a la implementación del SAAC. La familia se siente responsable del desarrollo de su hijo, y tiene la necesidad de ser partícipe del mismo.

Por otra parte, el profesional del centro de Atención Temprana donde acude el niño con dificultades en la comunicación y el lenguaje, también afirma que existe una constante comunicación con 
la familia implicada. Afirma que están muy pendientes de los progresos de su hijo, y se preocupan por la situación que están atravesando. Declara que no todas las familias se encuentran tan implicadas como este caso y, como hemos visto en la bibliografía más arriba, esta participación puede ser el punto de inflexión que hacia distintas direcciones en el progreso y desarrollo del lenguaje de este niño.

El profesional asegura que se dan reuniones mensuales con la familia, y, además, también se tiene constancia de los pequeños logros que va alcanzando el pequeño de forma semanal, ya que en los momentos de encuentro a la entrada o la salida del centro, también existe una breve comunicación entre los implicados.

Finalmente, el maestro-tutor del niño asegura que existe una buena coordinación entre los tres ejes implicados en el estudio. No podemos olvidar el papel que juega este sujeto en el desarrollo del niño con problemas de comunicación, debido a que se trata de un pilar fundamental en la adquisición del lenguaje del niño. Es el maestro-tutor el que, en el aula de Educación Infantil del centro educativo regular al que asiste el niño, da las pautas y los procesos convenientes, junto con el resto de alumnos, para poder adquirir un lenguaje acorde con su edad, el cual irá desarrollando a lo largo de toda su vida.

El maestro asegura que se dan reuniones con los familiares de forma trimestral. Tal y como se ha estudiado en la bibliografía existente, es lo más común en el ámbito de la Atención Temprana. Sin embargo, es de vital importancia que se den reuniones más asiduamente cuando el alumno o alumna presenta problemas de comunicación, debido a que puede que se den cambios o progresos en el niño en mucho menos tiempo que tres meses, y la familia debe ser conocedora de todo el proceso que su hijo recorre a lo largo del curso escolar.

Además, tampoco se han dado reuniones presenciales con el profesional de AT, sino que se ha limitado a una comunicación vía email o telefónica. A pesar de que esta comunicación se da de forma más frecuente que las reuniones que se tienen con los progenitores participantes, puede que exista una falta de presencialidad en esta coordinación.

Por último, cabe resaltar que no se dan reuniones de los tres ejes principales implicados en el estudio, algo que se echa en falta, teniendo en cuenta que la coordinación debe darse en los tres sujetos implicados.

\subsubsection{Fase 2. Grupo de Discusión}

Participantes y Contexto. A diferencia del procedimiento anterior, donde prima el carácter de representatividad y la necesidad de que existiera una coordinación entre los distintos implicados participantes, en esta fase de la investigación prevalece la necesidad de obtener distintas opiniones sobre las prácticas centradas en la familia, la coordinación entre los distintos ejes que participan de nuestra 
investigación y las creencias sobre los sistemas de comunicación alternativos y/o aumentativos. El tipo de muestreo utilizado fue no probabilístico, intencional.

A la hora de escoger la muestra que participará en el grupo focal, en primer lugar, se hizo un filtro sobre las familias que acuden con su hijo o hija a un CDIAT con problemas de comunicación y lenguaje. Como se ha explicado con anterioridad en la bibliografía revisada, la Atención Temprana no cubre únicamente los problemas o trastornos que implican las dificultades en el desarrollo de la comunicación en niños, sin embargo, este estudio sí que abarca esta problemática, y es por ello que nos centramos en este tipo de familias. Es por ello que se siguió un método de muestreo no probabilístico, por accesibilidad.

Es por ello, que se tienen en cuenta una serie de criterios para la inclusión de determinados sujetos en el grupo focal. Comenzando por las familias que se encuentran en un proceso de intervención con una posible implementación de un SAAC, y cuentan con una experiencia superior a tres meses acudiendo al centro de AT. Este requisito es imprescindible, puesto que se tiene la intención de contar con participantes expertos en esta temática en el grupo de discusión, con el fin de poder llegar a un debate con argumentos consolidados, y se tenga el conocimiento o la experiencia suficiente para llevarlo a cabo.

Por otra parte, también participarán en este grupo de discusión aquellos profesionales del centro que trabajen con estos niños con problemas de comunicación, como pueden ser logopedas, psicólogos y foniatras.

Finalmente, también serán invitados al grupo de discusión profesionales de distintos centros educativos, públicos y concertado-privados, que trabajen con niños que presentan problemas de comunicación, como pueden ser maestros-tutores que tengan en sus aulas alumnos con estas características, psicólogos del centro o profesionales maestros de audición y lenguaje.

La muestra final participante en el estudio es de 14 personas en el grupo focal, doce de ellas eran mujeres y dos eran hombres.

A continuación se muestra una tabla con los datos sociodemográficos de la muestra participante para la realización del grupo focal.

Tabla 1. Características de los participantes según lugar de trabajo, titulación y sexo

\begin{tabular}{|c|c|c|c|}
\hline Experto & Agente implicado & $\begin{array}{l}\text { Titulación/Nivel } \\
\text { estudios }\end{array}$ & Sexo \\
\hline Moderadora & Centro público & Maestra & $\mathrm{M}$ \\
\hline Experto 2 & Centro concertado & Maestra & M \\
\hline Experto 3 & Centro público & Maestra AyL & M \\
\hline Experto 4 & Centro público & Maestra & M \\
\hline
\end{tabular}




\begin{tabular}{llll}
\hline Experto 5 & CDIAT & Psicóloga & $\mathrm{M}$ \\
Experto 6 & Familiar & FPB & $\mathrm{H}$ \\
Experto 7 & Familiar & FPB & $\mathrm{M}$ \\
Experto 8 & Centro concertado & Maestra & $\mathrm{M}$
\end{tabular}

$\begin{array}{lllr}\text { Experto } 9 & \text { CDIAT } & \text { Foniatra } & \mathrm{M} \\ \text { Experto } 10 & \text { CDIAT } & \text { Logopeda } & \mathrm{M} \\ \text { Experto } 11 & \text { Centro público } & \text { Maestro } & \mathrm{M} \\ \text { Experto 12/Cámara } & \text { Familiar } & \text { Universitarios } & \mathrm{M}\end{array}$

$\begin{array}{lll}\text { Experto } 13 & \text { Familiar } & \text { ESO }\end{array}$

$\begin{array}{lll}\text { Secretaria } & \text { Centro concertado } & \text { Psicólogo }\end{array}$

Fuente: Elaboración propia.

Tal y como podemos observar, la profesión predominante en el grupo de discusión es la de maestro, siendo 5 los participantes implicados con esta titulación. Asimismo, también participaron dos psicólogos, una maestra de AyL, un logopeda y un foniatra, además de 4 familiares de niños con problemas de comunicación, asistentes a un CDIAT y a un centro educativo regular.

A continuación, se muestra una figura donde queda reflejada la disposición final que se organizará para el grupo focal. De este modo, todos los participantes se ven las caras, y resultará más sencillo para realizar el debate y la grabación del mismo.

Diseño de la Intervención. Para la elaboración del procedimiento perteneciente a la fase 2 de nuestro estudio empírico, se va a utilizar un grupo focal o grupo de discusión. Este tipo de procedimiento puede resultar útil, ya que permite explorar los conocimientos y opiniones acerca de las experiencias que poseen expertos en la temática a tratar, de un modo que incita a la interacción entre distintas personas con pensamientos, técnicas de trabajo y experiencias diferentes. Para ello, se han elaborado ad hoc una serie de preguntas guía (Anexo 2) con el fin de dirigir y encauzar el debate entre las distintas personas implicadas. Estas preguntas guía están orientadas a dar respuesta a los objetivos de nuestro informe de investigación, es decir, comprobar el nivel de interacción que se da entre los distintos grupos implicados, así como la calidad de esta comunicación y las ventajas o inconvenientes que se pueden dar en dicha coordinación durante el proceso de intervención para la implementación de un sistema de comunicación alternativo y/o aumentativo.

Como hemos visto con anterioridad, la técnica del Grupo Focal nos permitirá comprobar la disparidad de opiniones, percepciones y experiencias que tienen los expertos invitados al debate sobre 
esta coordinación, y los datos recogidos nos servirán más tarde para poder recoger, desde un enfoque cuantitativo, información sobre esta coordinación de la que hablábamos más arriba, y lograr así un mayor conocimiento sobre las posibles necesidades que puedan presentar estos colectivos con respecto a comunicación de familias, profesionales y maestros tutores en cuanto a las intervenciones de niños con problemas de comunicación y la posible implementación de sistemas de comunicación alternativos y/o aumentativos, para propiciar el desarrollo del lenguaje del infante.

Con motivo de la situación de pandemia que estamos viviendo en estos momentos, se siguió un protocolo para realizar el proceso de selección de los individuos que fueron invitados a participar en el grupo focal. A continuación se muestra una figura que resume el proceso de selección que se llevó a cabo.

Como podemos ver en la figura anterior, en un primer lugar, se informa a la muestra invitada por vía telefónica, explicando en qué consiste el estudio empírico y el por qué se hace necesaria su participación. Asimismo, se le envía a cada participante una carta de invitación (Anexo 3) vía email con información más detallada, y otros estudios realizados con anterioridad relacionados con la misma temática. Tras esto, la muestra participante debe confirmar su asistencia, con el objetivo de poder enviarles toda la información acerca del proyecto. Una vez confirmados los participantes, se realiza una última llamada telefónica para agradecer su participación y confirmar el lugar y el horario que tendrá el encuentro. Por último, procedemos a la presentación de los participantes en el grupo focal y al inicio del mismo.

Recogida de Información: Instrumentos y Procedimientos. Para la realización del grupo focal, se han elaborado una serie de preguntas guía que servirán de apoyo al entrevistador a la hora de dar paso al debate abierto entre los participantes. Se cuenta con la presencia explícita del entrevistador durante toda la duración del grupo focal.

Este grupo de discusión será realizado de forma presencial, cuidando las medidas de seguridad para el protocolo de Covid19, de modo que se disfrute de un entorno seguro y agradable. De ser posible, el grupo focal será realizado durante la tercera semana del trabajo de investigación, con la intención de obtener los resultados y poder analizarlos para llevar a cabo el siguiente procedimiento, es decir, el cuestionario. En el grupo de discusión (Anexo 2), se muestran las instrucciones que se le dan a las personas entrevistadas, con el fin de que la intervención sea lo más fructífera posible.

$\mathrm{Al}$ inicio de la sesión, se dio una ronda de presentaciones y se hizo un pequeño resumen del estudio de investigación sobre la coordinación entre los diferentes agentes implicados, como son familias, profesionales y maestros-tutores en cuanto a las intervenciones de niños con problemas de comunicación y la posible implementación de sistemas de comunicación alternativos y/o aumentativos, para propiciar el desarrollo del lenguaje del infante. 
Se estima que la duración del grupo focal sea de una hora y media aproximadamente, aunque se contará con un margen de cuarenta minutos por si pudiera suceder cualquier incidente que imposibilite el comienzo del mismo a la hora estimada ${ }^{1}$.

Las entrevistas quedarán grabadas en forma de vídeo y de audio, a través de dos grabadoras de audio de la marca SONY IC Recorder, modelo ICD-UX200, con disco interno de 2GB y una cámara de video SONY Handycam Hard Disck Drive, full HD 1080, con disco duro interno de 60GB. Además, la secretaria contará con una hoja de anotaciones donde irá escribiendo temas de relevancia que puedan ir surgiendo durante la entrevista.

Finalmente, se ha de tener en cuenta la transcripción de los datos del estudio, que será realizada en la semana 4 del proyecto, así como el análisis de los resultados del mismo, con el fin de obtener unos datos que nos sean de utilidad para la elaboración de un cuestionario en la parte cuantitativa de nuestro artículo de investigación, con el fin de poder obtener una mayor información acerca de la coordinación existente entre los distintos agentes implicados a la hora de la intervención con un niño que presenta problemas o dificultades en la comunicación y el lenguaje, y la posible implementación de un SAAC.

Plan de Tratamiento y Análisis de los Resultados. Como hemos planteado en la primera fase con la entrevista, con el fin de agilizar el proceso de análisis de la información obtenida, se utilizaría el programa Atlas.ti, ya que se trata de una herramienta muy útil a la hora de analizar la información de investigaciones de tipo cualitativo.

En primer lugar, realizaremos la transcripción de las grabaciones que hemos ido recopilando a lo largo del grupo de discusión, y las completaremos con las anotaciones que realizó la secretaria en la hoja de registro, en base a cuál ha sido su percepción sobre los temas más repetidos, en qué momento se han dado puntos de inflexión, entre otros.

Una vez transcritas las tres entrevistas realizadas, procederemos a introducir los datos al programa informático. El procedimiento que seguiremos será parecido al que utilizamos en análisis de datos de la entrevista, pero con la diferencia de que, en este caso, son muchos más los expertos que interactúan en el debate, por lo que tenemos más información y más datos que analizar. En este caso, es cuando nos será de mayor utilidad aún el haber escogido el software informático Atlas.ti, ya que nos permitirá organizar la información de modo que nos sea mucho más sencillo realizar el análisis.

Cabe resaltar que, de los resultados obtenidos en este procedimiento, se elaborará el cuestionario que se ha mencionado con anterioridad, el cual está orientado a dar respuesta, de una forma más amplia, a los objetivos que hemos planteado en este informe, es decir, analizar el nivel y la calidad de coordinación

\footnotetext{
${ }^{1}$ Nota: En el caso de que el estudio fuese llevado a cabo, se especificaría el lugar y hora exactos en los cuales se realizó el grupo de discusión.
} 
entre los centros de Atención Temprana, los centros de Educación Infantil y las familias que tienen un niño con problemas de comunicación para cuya mejora puede ser conveniente implementar un sistema aumentativo de comunicación.

En primer lugar, crearemos una nube de palabras que represente los términos que más se repiten durante el debate. Tras esto, se extraerán los conceptos o tópicos que más se repiten a lo largo del debate, de modo que creemos en el programa unos códigos que más tarde nos permitirán enlazar toda la información, y así poder ver las conexiones que existen entre las distintas opiniones y percepciones. Finalmente, se procederá a crear las redes, en función de los objetivos de investigación en las cuales están basadas las preguntas guía, y hemos especificado con anterioridad. De este modo, se podrá examinar con facilidad qué opina cada interlocutor con respecto a los tópicos más repetidos durante el grupo focal y se podrán analizar con exactitud los resultados obtenidos.

Resultados. Previo a comenzar con la exposición de los resultados obtenidos tras la puesta en práctica del grupo de discusión, cabe resaltar que este informe de investigación se trata de un estudio preliminar, por lo que se trata de una investigación ficticia. Es por ello que no ha sido posible recoger unos datos reales acerca del grupo de discusión planteado, el cual tiene unas preguntas guía ad hoc para dicho estudio. Por ello, se ha de recordar que los resultados que se exponen a continuación son únicamente una redacción de los posibles datos que podríamos haber obtenido si este se hubiera llevado a cabo realmente, en cuanto a la opinión de distintos expertos acerca de coordinación entre los implicados en el proceso a la hora de implementar un sistema de comunicación alternativo y/o aumentativo.

Tras realizar la transcripción de la información que hemos obtenido a partir de los datos proporcionados por los participantes del estudio, podemos decir que, en general, predomina la idea de que la coordinación entre los distintos implicados en el proceso a la hora de implementar un sistema de comunicación alternativo y/o aumentativo es necesaria, y trae consigo ventajas y beneficios para el niño que se encuentra en intervención. Sin embargo, existen disparidad de opiniones acerca de qué características debe tener esta coordinación entre colectivos.

Por un lado, la gran parte de profesionales pertenecientes a un CDIAT afirma que la participación de la familia es crucial en procesos de esta índole, ya que, como se ha mencionado con anterioridad, forman parte del día a día del infante y se encuentran en su entorno más cercano.

Por otro lado, también se declara por parte de este colectivo que muchas veces es complicado coincidir con familias o maestros para tener encuentros donde se pueda dar una conversación profunda acerca de las necesidades y progresos que va presentando el niño. Según argumentan, esto suele ser por falta de tiempo por parte de los otros dos colectivos. La gran mayoría de reuniones de esta índole se realizan cada dos o tres meses. 
Sin embargo, algunos de los familiares presentes en el grupo de discusión se opusieron a esta afirmación, argumentando que siempre que sea un problema inminente y de relevancia, se puede lograr el acuerdo para los encuentros semanales.

Del otro lado, los maestros opinan que, entre las horas lectivas y no lectivas que tienen, muchas veces les es muy complicado el poder concertar citas con los profesionales del CDIAT, y afirman que los procedimientos de comunicación más usados con el teléfono o el correo electrónico, tal y como veíamos en la primera fase de este trabajo.

Asimismo, los tres colectivos afirman que es esencial que los tres se encuentren inmersos en la misma atmósfera a la hora de poner en práctica una intervención de este tipo, y que en la balanza ganan las ventajas frente a los inconvenientes a la hora de organizar encuentros sobre la intervención y posible puesta en práctica de un SAAC a niños con problemas de comunicación.

Los profesionales de Atención Temprana afirman que, no se debe poner límites a la implicación de los padres o los maestros-tutores en estas intervenciones, sin embargo, señalan que es de vital importancia que estas personas se encuentren al margen mientras se realiza alguna intervención con el niño en el CDIAT. Es importante que los padres estén presentes, pero deben evitar distraer al infante mientras están trabajando con él, puesto que una figura familiar llamará su atención y podría descentrarle.

En esta misma línea, los profesionales del CDIAT apoyan que se dé una breve formación a los padres y maestros-tutores de los niños, de modo que estos conozcan el concepto de Atención Temprana, los distintos servicios que existen, y comprendan en todo momento en qué consiste la intervención la cual ellos también son partícipes y, de hecho, pilares fundamentales para que la misma resulte exitosa.

Finalmente, los tres colectivos afirmaron que, por motivo de la pandemia, estas reuniones han pasado de ser presenciales a ser por videollamada u otras técnicas que posibiliten la comunicación sin requerir la presencialidad de los sujetos implicados. Sin embargo, los profesionales del CDIAT argumentan que, con las medidas adecuadas, es posible realizar los encuentros que se hacían antes de la pandemia, con el objetivo de que, principalmente las familias puedan presenciar la intervención y las técnicas que el profesional utiliza con el niño en todo momento, ya que esto les servirá de ayuda para continuar con el proceso desde sus hogares.

Discusión de los Resultados. Si bien recordamos de la bibliografía analizada con anterioridad, es de vital importancia que se de esta comunicación entre padres, profesionales del CDIAT y maestrostutores a la hora de realizar una intervención a un niño con necesidades de Atención Temprana y la posibilidad de implementar un sistema de comunicación alternativo y/o aumentativo.

Como se ha podido comprobar, tras el análisis de los resultados obtenidos en este grupo de discusión, las reuniones entre estos tres colectivos no suelen ser tan frecuentes como las vistas en las 
entrevistas realizadas en la primera fase de nuestro estudio, sino que suelen darse cada cierto tiempo, de media cada dos o tres meses desde que comienza el proceso de intervención hasta que finaliza o termina el curso escolar. Sin embargo, se mantiene el contacto a través de correos electrónicos o vía telefónica.

Como bien explica la bibliografía utilizada, se dan muchos inconvenientes a la hora de realizar dichas reuniones, ya sea por falta de tiempo de los implicados, por falta de interés, o que se den ambas situaciones simultáneamente.

De hecho, las familias deberían ser el principal objetivo de la intervención, junto con el niño, puesto que es su entorno más cercano y se pretende que el programa de intervención no quede aislado al ambiente del CDIAT, sino que se extrapole a todos los ámbitos de la vida del niño. Es por ello que, además, es conveniente que las familias sean capaces de tomar sus propias decisiones, con la consulta del profesional de AT, para que puedan llegar a ser independientes del centro y consigan ser autosuficientes cuando la etapa de Atención Temprana del niño finalice. Por lo tanto, en ningún momento se deben poner límites a la implicación de los padres, o incluso los maestros-tutores, puesto que una mayor implicación de estos agentes siempre supondrá una mejora de los resultados obtenidos, así como un mejor desarrollo de los procesos de intervención.

En cuanto a la formación de los padres o maestros-tutores, con el fin de que conozcan unas nociones básicas en cuanto a la Atención Temprana, de modo que conozcan qué es lo que se está haciendo en todo momento durante la intervención con el niño que presenta problemas de comunicación. Por un lado, los profesionales de AT coinciden en que sería de gran ayuda este tipo de formación para los colectivos que no son profesionales de esta área. De hecho, si examinamos bien la bibliografía, podemos comprobar que se habla de una formación autodirigida, una motivación intrínseca por parte de los progenitores por aprender nociones básicas sobre el área de AT. Sin embargo, una problemática que también afecta a esto es, al igual que en los encuentros con los profesionales, la falta de tiempo o de interés que puede darse de forma aislada o conjunta.

Finalmente, la situación de Covid19 tiene las dos caras de la moneda. Por un lado, existe la posibilidad de que, al poder realizar reuniones por videollamada, los progenitores o maestros-tutores puedan extraer unas horas de su tiempo para dedicarlas a dichos encuentros, ya que no supone desplazarse ni se estima tanto tiempo como en reuniones presenciales. Sin embargo, por este mismo motivo, si los padres no disponen de los conocimientos necesarios de tecnología, puede ser un inconveniente y una posible causa de abandono de la motivación o el interés de los padres. Por otro lado, el miedo o la inseguridad que existe hoy en día por la situación de pandemia que vivimos, también puede dar lugar a la reticencia de estas personas a la hora de acordar encuentros presenciales con los profesionales del CDIAT o los maestros-tutores. 


\subsubsection{FASE 3. CUESTIONARIO}

Participantes y Contexto. Para realizar el muestreo de esta última fase cuantitativa de este proyecto, se ha escogido un método no probabilístico por conveniencia, ya que en nuestra muestra nos interesa a tener sujetos con unas determinadas características, como son profesionales de CDIATs que atienden a niños con problemas de comunicación, familias de niños con problemas de comunicación y maestros-tutores o maestros de Audición y Lenguaje que tengan o hayan tenido en sus aulas alumnos con estas características.

Se decidió aplicar este cuestionario a esta muestra, con el fin de poder generalizar los resultados a una población mayor, y conseguir la persecución de los objetivos de este estudio, que, si bien recordamos, se resumen en el objetivo principal, el cual pretende analizar el nivel y la calidad de coordinación entre los centros de Atención Temprana, los centros de Educación Infantil y las familias que tienen un niño con problemas de comunicación para cuya mejora puede ser conveniente implementar un sistema aumentativo de comunicación.

Con el fin de que el cuestionario llegara al mayor rango de personas de nuestra población posible, se siguieron una serie de procedimientos para hacerles llegar la encuesta. En primer lugar, se envió un correo electrónico a todos los CDIAT de la Región de Murcia y centros educativos públicos y concertadoprivados, ofreciéndoles la participación en este estudio, y mostrándoles una breve descripción de en qué consistía esta encuesta, y el proyecto de investigación. Por motivos de privacidad, en este primer rastreo no se pudo enviar este email a los progenitores de niños con problemas de comunicación asistentes a un CDIAT, por lo que solicitamos a los profesionales de los centros que transmitieran la intención de hacer partícipes a los padres implicados en estos procesos de intervención, con el objetivo de conseguir la mayor muestra representativa posible.

Tras la respuesta de la muestra invitada, se le envió a la muestra participante otro documento con más detalles acerca del proyecto de investigación, del cual van a ser partícipes, así como el cuestionario a cumplimentar.

La muestra final consta entre unos 60 y 80 cuestionarios cumplimentados.

A continuación se muestra una tabla con los datos sociodemográficos ${ }^{2}$ de la muestra participante para la realización del cuestionario

\footnotetext{
${ }^{2}$ Nota: En el caso de haberse llevado a cabo este procedimiento, se habría añadido los valores reales de la muestra. Sin embargo, al tratarse de una muestra ficticia, he preferido realizarlo de este modo.
} 
Tabla 2. Características sociodemográficas de los participantes según edad, sexo, nivel de estudios y ocupación

\begin{tabular}{|c|c|c|c|c|c|c|c|c|c|c|c|}
\hline Colectivo & Edad & & $\begin{array}{l}\text { Se } \\
(\%\end{array}$ & & Nivel de es & dios & & Ocupació & & & \\
\hline & $\begin{array}{l}\text { Medi } \\
\text { a }\end{array}$ & $\begin{array}{l}\text { Rang } \\
\mathrm{o}\end{array}$ & $\mathrm{H}$ & $\mathrm{M}$ & $\begin{array}{l}\text { Títulos } \\
\text { universitar } \\
\text { ios }\end{array}$ & $\begin{array}{l}\text { FP } \\
\text { Bachillera } \\
\text { to }\end{array}$ & $\begin{array}{l}\mathrm{ES} \\
\mathrm{O}\end{array}$ & $\begin{array}{l}\text { Psicólogo } \\
\text { S }\end{array}$ & $\begin{array}{l}\text { Logopeda } \\
\text { S }\end{array}$ & $\begin{array}{l}\text { Maestro } \\
\mathrm{S}\end{array}$ & $\begin{array}{l}\text { Otro } \\
\mathrm{s}\end{array}$ \\
\hline $\begin{array}{l}\text { Profesional } \\
\text { es CDIAT }\end{array}$ & $\mathrm{x}$ & $\mathrm{X}$ & $\mathrm{X}$ & $\mathrm{x}$ & $\mathrm{x}$ & $\mathrm{X}$ & $\mathrm{x}$ & $\mathrm{X}$ & $\mathrm{x}$ & $\mathrm{x}$ & $\mathrm{x}$ \\
\hline Familiares & $\mathrm{x}$ & $\mathrm{x}$ & $\mathrm{x}$ & $\mathrm{x}$ & $\mathrm{x}$ & $\mathrm{x}$ & $\mathrm{x}$ & $\mathrm{x}$ & $\mathrm{x}$ & $\mathrm{x}$ & $\mathrm{x}$ \\
\hline $\begin{array}{l}\text { Prof. } \\
\text { Centros } \\
\text { educativos }\end{array}$ & $\mathrm{x}$ & $\mathrm{x}$ & $\mathrm{x}$ & $\mathrm{x}$ & $\mathrm{x}$ & $\mathrm{X}$ & $\mathrm{x}$ & $\mathrm{x}$ & $\mathrm{x}$ & $\mathrm{x}$ & $\mathrm{x}$ \\
\hline
\end{tabular}

Fuente: Elaboración propia.

Diseño de la Intervención. Para la elaboración del procedimiento de la fase 3 de nuestro proyecto, la fase cuantitativa, se va a elaborar un cuestionario ad hoc, el cual llevará por nombre Cuestionario de Actitudes hacia la coordinación entre los distintos agentes implicados en una intervención en AT con implementación de un $S A A C$. Este procedimiento permite obtener datos sobre una temática determinada desde una muestra mucho más amplia que los dos procesos utilizados anteriormente. Es por ello que este cuestionario se realiza con los resultados obtenidos en la parte de enfoque cualitativo de este estudio.

Con este cuestionario, pretendemos llegar a la mayor población posible, con el fin de identificar el nivel y la calidad de coordinación que se da en los distintos CDIATs de la Región de Murcia, con respecto a las familias y otros profesionales inmersos en intervenciones en niños con problemas de comunicación y con posibilidad de implementación de un SAAC, y la opinión que tienen los participantes acerca de su experiencia con o sin esta coordinación, así como las ventajas e inconvenientes que puede dar esta existencia o ausencia de coordinación.

Recogida de Información: Instrumentos y Procedimientos. En primer lugar, para la elaboración de los ítems correspondientes a este cuestionario, se ha analizado la información obtenida en la segunda fase de nuestro proyecto, correspondiente al grupo de discusión. Con los resultados obtenidos en esta prueba, confeccionaremos un cuestionario ad hoc, con el fin de conocer las percepciones que tiene la muestra planteada anteriormente acerca de la coordinación que se da en los distintos CDIATs de la Región de Murcia, con respecto a las familias y otros profesionales inmersos en intervenciones en niños con problemas de comunicación y con posibilidad de implementación de un SAAC. 
En primer lugar, se elaborarán las preguntas en función del objetivo principal y los diferentes objetivos específicos de los que consta nuestro informe, ya que su fin último es la persecución de dichos objetivos. En cada una de estas preguntas, se solicita al participante que valore del 1 al 5 cada uno de los temas propuestos en este cuestionario, en una escala Likert (siendo 1 totalmente en desacuerdo y 5 totalmente de acuerdo).

Realizando una estimación, el cuestionario constaría alrededor de unos 20 ítems, parte de ellos haciendo referencia al nivel y la calidad de la coordinación que se da en los distintos CDIATs de la Región de Murcia, con respecto a las familias y otros profesionales inmersos en intervenciones en niños con problemas de comunicación y con posibilidad de implementación de un SAAC, otra parte a la opinión que tienen los encuestados sobre la formación para padres y maestros en términos de Atención Temprana, y otra a la experiencia propia de cada uno de los encuestados acerca de la coordinación que han percibido en posibles situaciones similares que hayan vivido con anterioridad. En los tres casos se les pedía a los participantes que valoraran del 1 al 5 su acuerdo o desacuerdo con las afirmaciones expuestas en el cuestionario.

Previo a la finalización de este cuestionario, este debe ser validado por un juicio de expertos, con el propósito de obtener garantías de rigor científico del instrumento. Según Escobar-Pérez y CuervoMartínez (2008), el juicio de expertos es "una opinión informada de personas con trayectoria en el tema, que son reconocidas por otros como expertos cualificados en éste, y que pueden dar información, evidencia, juicios y valoraciones." (p. 29).

El objetivo principal del juicio de expertos se basa en validar contenido en una prueba diseñada ad hoc para este trabajo de investigación. En el Anexo 4, documento el cual será facilitado a los expertos que procederán a la validación del instrumento, se especifican tanto las dimensiones como los indicadores que está midiendo cada uno de los ítems de la prueba. También aparece el diseño de las planillas para la validación de cada uno de los ítems.

Este juicio de expertos contará con, aproximadamente 8 expertos que validen el cuestionario realizado ad hoc. Entre los expertos seleccionados podremos encontrar profesores del departamento de Psicología Evolutiva y de la Educación de la Universidad de Murcia, maestros de Educación Infantil y profesionales de distintos CDIATs de la Región.

A estos profesionales se les proporcionará un documento (Anexo 4) con el fin de que evalúen finalmente la consistencia y validez del instrumento elaborado, teniendo en cuenta algunos criterios de validación, como pueden ser, según Serrano-Pastor (2020):

- Pertinencia: Grado en el que la dimensión se ajusta al objeto de estudio. 
- Adecuación: Grado de relación lógica o vínculo entre la dimensión y el constructo teórico que se evalúa.

- Suficiencia: Grado en el que los ítems que pertenecen a una misma dimensión bastan para obtener la medición de ésta.

- Coherencia: Grado de vínculo entre el ítem y la dimensión a la que pertenece

- Relevancia: o Importancia Grado en el que el ítem es esencial o importante en relación al resto de ítems de la dimensión, es decir, debe ser incluido

- Claridad: Grado en el que el ítem se comprende fácilmente para los destinatarios (p. 11).

Por último, para calcular la concordancia de las validaciones del juicio de expertos, se utilizará el coeficiente $\mathrm{W}$ de Kendall, que revelará si finalmente existe concordancia estadísticamente significativa o no entre los rangos promedios asignados por los jueces en relación a la relevancia de los ítems $(\mathrm{p}<0.5$ o $\mathrm{p}>0.5$ respectivamente).

Además, también debemos tener en cuenta la intensidad de la concordancia de dichos jueces. Teniendo en cuenta los valores de Landis y Koch (1977), el resultado que nos dé la W de Kendall representará los siguientes valores:

Tabla 3.Valores W de Kendall según Landis y Koch (1977)

\begin{tabular}{ll}
\hline Valor W de Kendall & Grado de acuerdo \\
\hline .00 a .20 & Pobre \\
.21 a .40 & Leve \\
.41 a .60 & Moderado \\
.61 a .80 & Substancial \\
.81 a 1.00 & Casi perfecto \\
\hline
\end{tabular}

Fuente: Elaboración propia.

Tras la obtención de los resultados de la validación del juicio de expertos, realizaremos las modificaciones pertinentes, siguiendo las indicaciones que nos han dado los expertos.

Tras esto, pasaremos el cuestionario, en un primer momento, a una muestra más pequeña, similar a la muestra real que ha sido seleccionada con anterioridad, con el propósito de realizar un estudio piloto, para tener la certeza de que nuestro instrumento es válido y es de interés realizar la aplicación a la muestra real, con el fin de obtener unos resultados fiables.

Por último, se realizará la aplicación final del cuestionario a la muestra seleccionada. Una vez que obtengamos los datos, utilizaremos el programa SPSS para el análisis de la información, así como la interpretación ${ }^{3}$ de los datos obtenidos.

\footnotetext{
${ }^{3}$ Nota: En el caso de haber llevado a cabo la investigación, este cuestionario habría sido elaborado, y habríamos obtenido unos resultados para interpretarlos. Por la situación de pandemia que estamos atravesando, no ha sido posible, por lo que el estudio se ha limitado al planteamiento y explicación de los procesos que se habrían seguido en su caso.
} 
A continuación, se muestra esquema que resume los pasos seguidos a la hora de la elaboración y puesta en práctica del procedimiento de enfoque cuantitativo, es decir, el cuestionario.

\section{CONCLUSIONES}

\subsection{CONCLUSIONES GENERALES}

A lo largo de este estudio de investigación, se ha mostrado la necesidad y la importancia de que exista una buena coordinación entre los agentes implicados en una intervención en el ámbito de la Atención Temprana.

Tras la elaboración de este estudio, resulta inminente que se dé una coordinación entre los distintos grupos implicados en estas intervenciones, para poder extrapolar las actuaciones que se dan en un CDIAT a lugares de confianza para el pequeño. Tal y como hemos visto en la bibliografía revisada, existen varios modelos de coordinación de familias con el CDIAT, pero destacan dos en concreto: el modelo de padres co-terapeutas y el modelo de Intervención Centrada en la Familia.

Además, también es importante resaltar el principal motivo por el cual los niños con necesidades de Atención Temprana asisten a estos centros. La gran mayoría de estos niños presentan problemas en la comunicación y el lenguaje oral, y estos problemas pueden darse como casos aislados, o acompañados de algún trastorno, discapacidad o enfermedad que los acompañe o los provoque.

Por lo tanto, se decidió encaminar este informe de investigación a la concordancia que existe o no entre los distintos agentes implicados en estas intervenciones en niños con problemas de comunicación y lenguaje oral. Además, también se quiso incluir en esta investigación a los maestros-tutores de estos niños, con el motivo de la escasa información que se encuentra acerca de estos sujetos como co-protagonistas de los procesos de intervención en este ámbito.

Sin embargo, la situación de Covid19 por la cual estamos pasando impidió llevar a cabo la investigación de un modo verídico, por lo que este artículo se ha limitado al planteamiento de los procedimientos que se habrían utilizado en el caso de haber sido posible la puesta en práctica de la investigación, así como a la conjetura de los datos que habríamos obtenido en la parte de este trabajo perteneciente al enfoque cualitativo, con la intención de poder extraer unas conclusiones ficticias de lo que se ha obtenido finalmente.

Es por ello, que, en base a los objetivos de investigación de este artículo y de la bibliografía revisada, se redactarán a continuación una serie de conclusiones que se han obtenido tras el análisis y estudio de la información obtenida en este proyecto empírico:

- En primer lugar, cabe destacar que el principal foco de la Atención Temprana en nuestros días no es otro que el niño y el entorno que le rodea, en todo su conjunto. 
Al realizar intervenciones en el niño con necesidades de Atención Temprana, y más concretamente, con problemas de comunicación, debemos contar, además, con todos los agentes implicados en su día a día, como lo son la familia y, en su caso, el maestro tutor.

- En función del objetivo principal, el cual se basa en analizar el nivel y la calidad de coordinación entre los centros de Atención Temprana, los centros de Educación Infantil y las familias que tienen un niño con problemas de comunicación para cuya mejora puede ser conveniente implementar un sistema aumentativo de comunicación, se puede decir que sí se da una coordinación en la mayor parte de los casos analizados. Sin embargo, muchas veces, esta coordinación no es lo suficientemente constante y periódica.

Por lo tanto, podemos concluir que, a pesar de que se da esta coordinación entre los distintos agentes implicados en el proyecto, estas reuniones o encuentros deberían darse con más frecuencia, con el propósito de conocer en todo momento las necesidades y progresos del niño que presenta problemas de comunicación y al cual se está implementando un SAAC.

- En relación al objetivo que habla sobre la valoración de la coordinación entre los agentes implicados a través de métodos de enfoque cualitativo, se ha realizado una aproximación a los posibles resultados que podríamos haber obtenido de estos procedimientos, ya que, por la situación actual de pandemia, no ha sido posible llevarlo a cabo de forma real.

Sin embargo, basándonos en esta aproximación, se puede concluir que los profesionales tienen una opinión positiva acerca de que se dé una coordinación entre los distintos implicados en los procesos de intervención, a pesar de que esta sea escasa. También coinciden en que sería de gran utilidad la formación para padres y maestros sobre nociones básicas de la Atención Temprana, o, en este caso, sobre los problemas de comunicación que sufren los niños que están siendo intervenidos. 


\section{REFERENCIAS}

Cabello-Luque, F., \& Mazón-Morillas, C. (2018). Iconicidad y facilidad de aprendizaje de los símbolos pictográficos ARASAAC. Revista de Logopedia, Foniatría y Audiología, 38(3), 95104. doi:10.1016/j.rlfa.2018.04.002

Escobar-Pérez, J. \& Cuervo-Martínez, A. (2008). Validez de contenido y juicio de expertos: Una aproximación a su utilización. Avances en Medición, 6, 27-36.

Fortea, M. S. et al. (2015): Desarrollo temprano del lenguaje en niños pequeños con trastorno del espectro autista mediante el uso de sistemas alternativos. Revista de Neurología, 60 (Supl.1):31-35.

García, M. P., Madrid, D., \& Galante, R. (2017). Children and Augmentative or Alternative Communication System (AACs): a perceptive vision of the role played by families and professionals. Anales de Psicología, 33(2), 334-341. https://dx.doi.org/10.6018/analesps.33.2.267631

Grupo de Atención Temprana (GAT) (2000). Libro Blanco de la Atención Temprana. Madrid: Ministerio de Trabajo y Servicios Sociales. Real Patronato de Prevención y de Atención a Personas con Minusvalía. Knowles, M. (1984). Andragogy in action. San Francisco, SA: Jossey-Bass.

Landis J.R. \& Koch G.G. (1977) The measurement of observer agreement for categorical data. Biometrics 33,159-174.

McDonough, D. (2013). Similarities and Differences between Adult and Child Learners as Participants in the Natural Learning Process. Psychology, 4, 345-348. doi: 10.4236/psych.2013.43A050.

Muñoz, J. \& Sahagun, M. (2017). Hacer análisis cualitativo con Atlas.ti 7. Manual de uso. Recuperado de https://manualatlas.psicologiasocial.eu/atlasti7.pdf

Novak, I. \& Honan, I. (2019). Effectivenes of paediatric occupational therapy for children with disabilities: A systematic review. Australian Occupational Therapy Journal, doi: 10.1111/14401630.12573

Novak, I., Mcintyre, S., Morgan, C., Campbell, L., Dark, L., Morton, N., et al. (2013). A systematic review of interventions for children with cerebral palsy: the state of the evidence. Developmental Medicine \& Child Neurology, 55(10), 885-910. http://doi.org/10.1111/dmcn.12246

Novak, I., Morgan, C., Fahey, M., Finch-Edmondson, M., Galea, C., Hines, A., et al. (2020). State of the Evidence Traffic Lights 2019: Systematic Review of Interventions for Preventing and Treating Children with Cerebral Palsy, 1-21. http://doi.org/10.1007/s11910-020-1022-z

Serrano-Pastor, F. J. (2020, 17 de diciembre) Criterios de rigor científico de los instrumentos de recogida de información en la investigación educativa avanzada. Métodos Avanzados de Investigación Educativa Máster Universitario en Investigación, Evaluación y Calidad en Educación Universidad de Murcia [presentación de diapositivas].

Serrano-Pastor, F.J. (2020, 15 de septiembre). Técnicas e instrumentos de recogida de información en la investigación educativa avanzada [presentación de diapositivas].

Tesauro de la Unesco. Recuperado de: http://vocabularies.unesco.org/browser/thesaurus/es/ 